\title{
Research on the Impact of Video-Based Native Ads on Users' Purchase Intention Under the Perceived Value Theory
}

\author{
MINGJIA Lin ${ }^{1}$, KAI Tang ${ }^{2}$, QINGJI Fan ${ }^{3 *}$
}

\begin{abstract}
${ }^{1}$ Business School, Yangzhou University, Yangzhou, Jiangsu, China
${ }^{2}$ Business School, Yangzhou University, Yangzhou, Jiangsu, China

${ }^{3}$ Business School, Yangzhou University, Yangzhou, Jiangsu, China

"Corresponding author. Email: qjfan@yzu.edu.cn
\end{abstract}

\begin{abstract}
In this era of information explosion, users tend to be bombarded with overloading information, which increasingly deteriorates the validity of traditional advertising. Fortunately, the emergence of native advertising, a brand-new form in this area, has effectively alleviated this dilemma. However, due to its rapid development, there still exist some unresolved problems. Based on the theory of perceived value, this paper analyzes the data by means of questionnaire survey, explores the impact of native video ads on users' purchasing intention and moves on to offer some suggestions for its development.
\end{abstract}

Keywords: perceived value, native ads, purchase intention

\section{感知价值理论下 \\ 视频类原生广告对用户购买意愿影响研究 \\ 林铭佳 ${ }^{1}$, 唐凯 ${ }^{2}$, 范庆基 ${ }^{3, *}$,}

\begin{abstract}
扬州大学商学院, 扬州, 江苏, 中国
?扬州大学商学院, 扬州, 江苏, 中国

扬州大学商学院, 扬州, 江苏, 中国

*通讯作者. 邮箱:qjfan@yzu.edu.cn
\end{abstract}

中文摘要:

在信息爆炸的当下，用户信息接受量严重超载，传统广告效益迅速衰减。而新的广告形式即原生广告 的出现有效地缓解了这一状况, 但由于其发展过快, 仍存在一定问题。本文基于感知价值理论, 采用 问卷调查的方式, 对数据进行分析, 探究视频类原生广告对用户购买意愿的影响, 为原生广告的发展 及提出建议。

关键词：感知价值，原生广告，购买意愿

\section{1. 引言}

在信息泛化的互联网时代, 用户对于广告的抵触情 绪达到前所未有的程度, 这使传统广告难以产生效果。 因此, 新的广告形式即原生广告的出现, 使得广告主所 传达的信息更容易被大众接收, 并能起到“广告即内容”
的效果。原生视频广告为企业产品、品牌营销提供了新 的解决方案, 目前短视频营销生态已经渐趋成熟, 用户 结构逐渐稳定，同时市场规模也逐年爆发式增长。然而 过快的发展往往会出现问题, 目前原生视频广告面临着 内容无法完全与应用匹配、广告质量良莠不齐、广告铺 陈时间较长等问题，在一定程度上影响用户的交互体 验, 削弱了广告效果。感知价值理论由 20 世纪 80 年代 
的营销学者提出, 如今感知价值理论已经广泛地运用在 移动电子商务、移动社交网络、在线平台、网络购物等 一系列特定情景之中, 成为营销领域的一个重要理论基 础。在目前对原生广告的研究中, 学者们广泛采用认知 模型、广告效果评估模型、信息传播模型等来解释与梳 理原生广告对于消费者影响的机理。顾客感知价值理论 能很好地阐释顾客感觉与知觉所形成的 “感知” 过程, 而消费者所 “感知” 的价值在很大程度上影响消费者消 费心理与行为变化过程, 以感知价值理论来研究顾客面 对原生广告所宣传产品与品牌的感知价值, 对于原生广 告本身的发展与广告效果的提升有较高的参考意义。

\section{2. 文献回顾}

\section{1 原生广告}

自 2012 年引入国内开始, 原生广告便快速发展, 2017 年进入繁荣时期, 国内外原生广告的研究与应用 随着时间的推移越发丰富, 不同学者探寻不同原生广告 的形式, 从不同角度展开研究探索。国外最早提出原生 广告概念的 Fred Wilson 认为 “原生广告是一种从网站 和用户出发的盈利模式, 由广告内容所驱动, 并整合了 网站和 app 本身的可视化设计” [1]。通过归纳总结学 者们的研究, 原生广告有如下特点: 1、原生广告的内 容、风格与形式多样化且与媒体十分贴合; 2、原生广 告更符合用户的兴趣与需要; 3、原生广告提升用户体 验, 并提供价值。因此我们认为原生广告是一种形式多 样化, 贴合用户使用环境和需求, 融入用户体验的广告 形式。

随着 $4 \mathrm{G}$ 移动网络的普及, 各类短视频 app 展开激 烈的角逐, 广告是各大短视频 app 核心盈利模式, 视频 类原生广告锋芒毕露。这类广告具有丰富的内容、出人 意料的创意, 在各大应用中频繁出现。原生广告不仅为 用户提供有价值的广告信息, 还提高了广告的曝光率、 转化率, 成为营销广告的新模式。视频类原生广告即采 用视频的形式, 在媒介内自动播放并且能自然地与页面 融合的广告类型。其主要的形式有信息流视频广告、创 意广告、定制视频广告等。因为其运用广泛、受众面广, 得到市场的追捧, 自 2015 年起市场规模呈现几何倍数 增长。然而, 视频类原生广告繁荣发展之下还潜藏着融 合程度不足、质量良莠不齐等问题亟待解决。

\section{2 感知价值理论}

在互联网时代, 感知价值具有较广的应用面, 并且 理论也进一步丰满以适用于新的领域。上个世纪 80 年 代的学者 Zeithaml 认为消费者购买某样商品或服务所 付出的不仅仅是货币, 还有时间、精力等, 这也会影响 消费者对于商品的感知价值。她认为感知价值是顾客对 所感知到的利益与其获得商品或服务时付出的成本进
行权衡后，对商品或服务效用的总体评价 [2]。在感知 价值的研究中, 汪涛等人基于心理账户的禀赋效应和支 付贬损来研究顾客参与对顾客感知价值的影响机制 [3] ; 王建军等探究网络口碑、感知价值对于购买意愿 的内部作用机理 [4]; 董大海、杨毅回顾了感知价值的 概念研究, 认为在网络环境下消费者感知价值来源于产 品本身与购买的情境, 并将消费者感知价值分为结果性 价值、程序性价值、和情感性价值 [5]。虽然感知价值 理论随时间不断丰满完善, 尤其在网络环境下的研究成 果近年来硕果累累，但是感知价值理论在广告方面的应 用实属有限，而应用在原生广告对于消费者购买意愿影 响因素的探究上尚未有所成果。因此本研究基于 Zeitham1 [2] 的感知价值理论, 探究用户的感知价值对 用户广告态度和购买意愿之间关系的影响作用。

\section{3. 研究模型与假设}

\section{1. 变量设置及假设}

本文的研究模型主要基于感知价值理论进行设计, 将原生广告形式下影响用户购买意愿的因素分为感知 收益、感知成本、社群影响、广告态度、购买意愿。

\section{1. 1. 感知收益}

在感知收益的相关研究中, 感知收益被认为是用户 感知到的广告效果为其提供有用信息、提高选购效率的 能力 [6]。王黎明等人其将感知收益分为享乐价值、功 能价值、体验价值, 结果证实了感知收益正向显著影响 移动端位置广告服务中用户采纳态度和采纳意愿 [7]。

因此, 结合原生广告的特性, 我们根据王黎明等 [7] 的 分类, 将原生广告的感知收益划分为: 享乐价值、功能 价值和体验价值。享乐价值指原生广告带给用户的乐 趣、享受等精神上的愉悦体验; 功能价值指原生广告提 供的内容完整度、丰富度、可靠性等; 体验价值指原生 广告带给用户便利与交互感。提出以下假设:

$\mathrm{H} 1$ : 原生广告的感知收益正向影响用户的广告态 度： 态度;

$\mathrm{H} 1 \mathrm{a}$ : 原生广告的享乐价值将正向影响用户的广告

$\mathrm{H} 1 \mathrm{~b}$ : 原生广告的功能价值将正向影响用户的广告 态度;

H1c: 原生广告的体验价值将正向影响用户的广告 态度;

\section{1.2. 感知成本}

感知成本又称感知付出, 是指用户在观看广告过程 中以及观看后所带来的财务、心理和时间的付出 [6]。 
有学者将感知成本作为一个总维度, 细分感知成本的各 维度, 如王黎明等人感知付出分为隐私关注和感知风 险, 发现感知付出负向影响用户采纳态度 [7]; 董庆兴 等人将感知成本划分为隐私安全性、反馈及时性, 发现 其负向影响在线健康社区用户满意度 $[8]$ 。结合原生广 告特性, 将感知成本分为感知时间成本、感知价格和感 知风险。感知时间成本定义为用户在观看原生广告时所 投入的时间长度; 感知价格定义为用户认为原生广告所 宣传的产品和服务实际价格偏高; 感知风险定义为用户 认为原生广告所宣传的产品或服务将夸大产品或服务 的效果, 导致用户期望和产品实际不符。

$\mathrm{H} 2$ : 原生广告的感知成本将负向影响用户的广告态 度:

$\mathrm{H} 2 \mathrm{a}$ : 原生广告的感知产品价格将负向影响用户的 广告态度;

$\mathrm{H} 2 \mathrm{~b}$ : 原生广告的感知时间成本将负向影响用户的 广告态度;

$\mathrm{H} 2 \mathrm{c}$ ：原生广告的感知风险将负向影响用户的广告 态度;

\section{1. 3. 社群影响}

社群影响是指用户所感受到的对原生广告的态度 受周围群体的影响程度 [6]。在以往的研究里, 有学者 将其作为感知价值的一个外部因素的维度, 如社会价值 [9]; 也有学者采用社会影响的大小来分析用户采纳意 愿的程度 [7]; 还有学者借助 UTAUT 模型探讨社群影响 对用户购买意愿或使用意愿的影响 [10]。因此在原生广 告研究中, 将社群影响定义为用户的家人或朋友对于原 生广告的态度、分享推荐的情况对于用户的影响。 告态度

H3: 原生广告带来的社群影响将正向影响用户的广

\section{1. 4. 广告态度与购买意愿}

影响消费者购买行为的因素多种多样, 广告只是其 中一个因素, 但是一个成功的广告应当对消费者的认 知、态度、行为产生影响 [11]。张望通过眼动仪研究用 户的故事性广告态度对购买意愿的影响, 发现广告态度 正向显著影响购买意愿 [11]。因此原生广告促进用户产 生积极的广告态度将会影响用户的购买意愿, 即用户在 观看原生广告后对广告持较好的态度, 并产生考虑购买 相关产品或服务的意向。 买意愿

$\mathrm{H} 4$ : 用户对原生广告的广告态度正向影响用户的购

\section{2. 数据收集}

本次调研采用线上问卷的形式, 问卷包含 9 个维度 以及人口统计学变量共 32 道题目。所有问项皆改编自
已有研究文献, 并在对人员进行访谈后, 基于原生广告 的特性进行了添补。研究采用李克特 7 级量表, 由 “ $1=$ 完全不同意” 到 “7=完全同意”。累计回收 229 份问卷, 剔除无效问卷后，共取得 212 份有效问卷，有效率 $92.58 \%$ 。其中受访者中男性 75 名, 女性 137 名, 年龄 段集中在 $15^{\sim} 30$ 岁, $86.3 \%$ 的受访者为本科学历, 都有 过观看视频类原生广告的经验。

\section{4. 数据分析及假设检验}

\section{1 数据分析}

利用 spss 25.0 和 amos 23 对 212 份数据进行信效度 分析、相关分析; 利用 amos 23 对数据进行验证性因子 分析 (CFA) 并建立结构方程模型 (SEM)。

各维度 Cronbach's Alpha 系数在 $0.616^{\sim} 0.842$ 之 间符合信度要求。经过相关性检验, 模型不存在多重共 线性问题，其各变量相关系数远低于建议阈值 0.7 , 可 以构建相应的结构方程模型。通过验证性因子分析剔除 效度较低的题项后, 计算收玫效度 AVE 值在 $0.45^{\sim} 0.77$ 之间, 符合量表效度要求; 计算组成信度 $\mathrm{CR}$ 值在 $0.624^{\sim} 0.849$ 之间, 符合量表组成信度要求。各构念的 AVE 平方根 (表 2 对角线) 皆大于构念间皮尔逊相关值, 因此具有区分效度。

表 1 样本数据信效度检验结果

\begin{tabular}{|c|c|c|c|c|c|c|c|c|c|}
\hline \multirow[t]{2}{*}{ 构念 } & \multirow[t]{2}{*}{ 题目 } & \multicolumn{4}{|c|}{ 参数显著性估计 } & \multirow{2}{*}{\begin{tabular}{|c|} 
因素负荷量 \\
Std. \\
\end{tabular}} & \multirow{2}{*}{\begin{tabular}{|c|} 
组成信度 \\
$C R$
\end{tabular}} & \multirow{2}{*}{\begin{tabular}{|c|} 
收敛效度 \\
AVE
\end{tabular}} & \multirow{2}{*}{$\begin{array}{l}\text { 信度 } \\
\text { Alpha }\end{array}$} \\
\hline & & Unstd. & S.E. & $t$-value & $P$ & & & & \\
\hline \multirow[t]{3}{*}{ 享乐价值 } & 享乐价值 3 & 1 & & & & 0.726 & 0.849 & 0.653 & 0.842 \\
\hline & 享乐价值 2 & 1.139 & 0.099 & 11.532 & $* * *$ & 0.883 & & & \\
\hline & 享乐价值 1 & 1.138 & 0.104 & 10.963 & $* * * *$ & 0.808 & & & \\
\hline \multirow[t]{3}{*}{ 功能价值 } & 功能价值 1 & 1 & & & & 0.747 & 0.828 & 0.617 & 0.823 \\
\hline & 功能价值 2 & 1.073 & 0.095 & 11. 358 & $* * * k$ & 0.85 & & & \\
\hline & 功能价值 4 & 0.992 & 0.095 & 10.394 & $* * * k$ & 0.756 & & & \\
\hline \multirow[t]{2}{*}{ 体验价值 } & 体验价值 1 & 1 & & & & 0.877 & 0.816 & 0.69 & 0.81 \\
\hline & 体验价值 2 & 0.901 & 0.085 & 10.65 & $* * *$ & 0.782 & & & \\
\hline \multirow[t]{2}{*}{ 社群影响 } & 社群影响 3 & 1 & & & & 0.862 & 0.835 & 0.717 & 0.83 \\
\hline & 社群影响 2 & 0.958 & 0.121 & 7. 892 & $* * *$ & 0.831 & & & \\
\hline \multirow{2}{*}{\begin{tabular}{|l|} 
感知产品 \\
价格 \\
\end{tabular}} & 感知产品价格 2 & & & & & 0.536 & 0.678 & 0.527 & 0.63 \\
\hline & 感知产品价格 1 & 1.599 & 0.466 & 3. 433 & $* * *$ & 0.875 & & & \\
\hline \multirow[t]{2}{*}{\begin{tabular}{|l|} 
感知时间 \\
成本
\end{tabular}} & 感知时间成本 1 & 1 & & & & 0.598 & 0.624 & 0.456 & 0.616 \\
\hline & 感知时间成本 3 & 1.186 & 0.22 & 5.39 & $* * *$ & 0.745 & & & \\
\hline \multirow[t]{3}{*}{ 感知风险 } & 感知风险 2 & 1 & & & & 0.836 & 0.818 & 0.603 & 0.81 \\
\hline & 感知风险 3 & 0.975 & 0.086 & 11.374 & $* * * k$ & 0.835 & & & \\
\hline & 感知风险 4 & 0.74 & 0.08 & 9. 228 & $* * *$ & 0.643 & & & \\
\hline \multirow[t]{3}{*}{ 广告态度 } & 广告态度 3 & 1 & & & & 0.714 & 0.793 & 0.563 & 0.78 \\
\hline & 广告态度 2 & 1.292 & 0.154 & 8. 417 & $* * * *$. & 0.855 & & & \\
\hline & 广告态度 1 & 0.991 & 0.118 & 8.372 & $* * * *$ & 0.669 & & & \\
\hline \multirow[t]{2}{*}{ 购买意愿 } & 购买意愿 3 & 1 & & & & 0.745 & 0.77 & 0.77 & 0.767 \\
\hline & 购买意愿 2 & 1.042 & 0.132 & 7.88 & $* * * *$ & 0.718 & & & \\
\hline
\end{tabular}




\section{表 2 区别效度分析}

\begin{tabular}{|c|c|c|c|c|c|c|c|c|c|c|}
\hline & $\mathrm{AVE}$ & 广告态度 & 购买意原 & 社群影响 & 感知风险 & 感知时间成本 & 感知产品价格 & 体验价值 & 功能价值 & 享乐价值 \\
\hline 广告态度 & .563 & .750 & & & & & & & & \\
\hline 购买意愿 & .770 & . 693 & .87 & & & & & & & \\
\hline 社群影响 & .717 & . 570 & .69 & .847 & & & & & & \\
\hline 感知风险 & .603 & -.183 & .02. & -.050 & $.7 \pi$ & & & & & \\
\hline 感知时间成本 & .456 & -.353 & .072 & .084 & .615 & .67 & & & & \\
\hline 感知产品价格 & .527 & .031 & .23 & .142 & .415 & .396 & .72 & & & \\
\hline 体验价值 & .690 & .567 & .555 & .471 & -.155 & -.082 & .108 & .831 & & \\
\hline 功能价值 & .617 & .510 & .57 & .424 & -.153 & -.069 & .03 & .704 & .786 & \\
\hline 享乐价值 & .653 & . 592 & .555 & .422 & -.224 & -.080 & .24 & .609 & .660 & .808 \\
\hline
\end{tabular}

\section{2 假设检验}

通过一系列修正指标对模型的修正、基于理论与符 合现实逻辑的修改后, 结构方程模型的各拟合指标较为 良好, 其中 CMIN/DF 值为 1.508 小于合格值 3.0、替代 性指标 RMSEA 值为 0.049 小于优良值 0.05 ; GFI $=0.896$ 、 AGFI $=0.854$ 皆大于合格值 $0.70 ;$ IFI $=0.955$ 、 $\mathrm{TLI}=0.941 、 \mathrm{CFI}=0.954$ 皆大于优良值 0.90 。因此, 该 模型拟合度良好, 能够较好地解释用户对视频类原生广 告广告态度以及购买意愿。

\section{表 3 模型验证与假设检验}

\begin{tabular}{|c|c|c|c|c|c|c|c|c|}
\hline 假设 & \multicolumn{3}{|c|}{ 回归路径 } & Std. & S.E. & C. R. & $\mathrm{P}$ & 检验结果 \\
\hline H1a & 广告态度 & $<-$ & 享乐价值 & 0.307 & 0.081 & 2. 822 & 0.005 & 显著 \\
\hline $\mathrm{H} 1 \mathrm{~b}$ & 广告态度 & $<-$ & 功能价值 & 0.074 & 0.092 & 0.636 & 0.525 & 不显著 \\
\hline H1c & 广告态度 & ২- & 体验价值 & 0.157 & 0.092 & 1.437 & 0.151 & 不显著 \\
\hline H2a & 广告态度 & «- & 感知产品价格 & -0.005 & 0.106 & -0.051 & 0.959 & 不显著 \\
\hline H $2 \mathrm{~b}$ & 广告态度 & $<-$ & 感知时间成本 & -0.403 & 0.098 & -3.095 & 0.002 & 显著 \\
\hline H $2 \mathrm{c}$ & 广告态度 & $<-$ & 感知风险 & 0.231 & 0.072 & 2. 165 & 0.03 & 显著 \\
\hline H3 & 广告态度 & $<-$ & 社群影响 & 0.465 & 0.065 & 5. 277 & 冰冰 & 显著 \\
\hline $\mathrm{H} 4$ & 购买意愿 & $<-$ & 广告态度 & 0.77 & 0.095 & 7. 718 & $* * * *$ & 显著 \\
\hline
\end{tabular}

根据最终修正模型的标准化回归路径系数图, 即功 能价值、体验价值、感知产品价格均不会显著影响用户 对于原生广告的态度, 因此假设 H1b、H1c、H2a 均不成 立。而感知风险显著正向影响广告态度, 与原假设相悖, 原假设 H2c 不成立。这可能由于用户对于原生广告的态 度比较积极, 并没有从中感受到风险。享乐价值、社群 影响显著正向影响广告态度, 因此假设 H1a、H3 成立。 感知时间成本显著负向影响广告态度, 因此假设 $\mathrm{H} 2 \mathrm{~b}$ 成 立。广告态度显著正向影响购买意愿, 因此假设 $\mathrm{H} 4$ 成 立。

\section{5. 研究结果与启示}

从数据分析结果来看, 原生广告中所提供信息的质 量、数量, 以及价格的可靠度对用户购买意愿的影响都 为不显著, 这说明随着国民经济的快速发展, 大众的消 费水平提高, 相应的消费观念也发生了改变, 对产品价 格的敏感度有所下降; 另外, 由于原生广告的结构特性, 其与产品相关的信息相对较少且不明显, 易被忽略, 对 购买意愿的影响较小。相较于产品信息, 用户更注重原 生广告带来的乐趣、享受等精神上的愉悦体验。

另一方面, 在信息爆炸的时代, 用户已经习惯了无
处不在的广告，所以原生广告出现的时间、地点是否恰 当对用户造成的影响较小且很容易被用户略过，而决定 用户是否会观看的因素是广告所呈现的第一印象，即广 告封面。据此，吸引用户眼球的封面设计是用户观看广 告的前提; 其次, 用户在观看、理解一则原生广告上花 费的时长会显著负向影响其购买意愿, 这符合当下快节 奏生活的社会状态，且据结果显示感知时间成本较享乐 价值而言对用户购买意愿的影响力更大, 所以长度适 中、易于理解的内容是用户感知享乐价值的前提。

研究还表明家人或朋友对原生广告的态度、看法会 影响用户观看该广告与产生购买动机的意愿; 广告态度 正向影响购买意愿, 良好的广告态度会促进产生购买意 愿。

根据上述研究结果及结论, 拟提出以下建议:

1. 确保原生广告封面设计的吸引力

信息泛滥的当下及快节奏的生活使得用户对信息 的篎选变得快速且随意, 更遑论广告, 视频类原生广告 要设计具有吸引力的封面, 为用户创造观看视频的动 力, 提高点击率, 这是广告内容得以传达出去的基础。

2. 确保原生广告内容的情节性

情节性更容易融入娱乐要素, 给用户带来精神上的 放松和愉悦, 显著改善其广告态度, 利于促进其购买意 愿; 另外, 情节性内容更易于广告时长的控制及产品的 融入, 富有情节性的内容不会让用户觉得冗长, 同时通 过联想利于客户理解、记住产品。

3. 确保原生广告传播的可分享性

移动端普及的当下，交互已成为各平台的必备功 能, 且为用户所需要, 所以原生广告在传播过程中要确 保可分享性，例如评论、转发等功能。一方面利于获取 用户对产品的主观反馈, 另一方面可以利于社群关系进 行传播、引导, 改善广告态度。

本文的局限于调查对象多以本科及大专在校生为 主, 且以女性居多, 未来研究者在进行数据收集时可以 增加样本的数量及不同年龄层面的覆盖度。

\section{REFERENCES}

[1] Ma Rui, Wang Ye. (2018) On Native Advertising under the New Media Environment[J].Journal of Hebei University of Economics and Business(Comprehensive Edition) 2018,18(04):13-17.

[2] ZEITHAM L V A. Consumer Perceptions of Price, Quality and Value: A Means-End Model and Synthesis of Evidence [ J]. Journal of Marketing, 1988, 52 (3): 2 22 .

[3] Wang Tao, Cui Nan and Yang Kui,The Effect of Customer Participation on Customer Perceived Value: Based on the Mental Accounting Theory[J].Journal of Business Economics,2009(11):81-88.

[4] Wang Jianjun, Wang Lingyu and Wang Meng meng,How eWOM and perceived value influence 
purchase intention: An investigation of mediation-moderation effects[J].Journal of Industrial Engineering and Engineering Management,2019,33(04):80-87.

[5] Dong Dahai and Yang Yi,Theoretic Analysis of Perceived Value by Consumers under Internet Environment[J].Chinese Journal of Management,2008(06):856-861.

[6] He Yuehong,An Empirical Research on the Influencing Factors of In-Feed Advertising from WeChat Moments[D].South China University of Technology,2018.

[7] Wang Liming, Liu Yanni and Qi Xiujing,Research on consumer adoption intention of mobile location advertising service based on user perceived value[J].Journal of Zhejiang Sci-Tech University,2019,42(8).

[8] Dong Qingxing, Zhou Xin, Mao Fenghua and Zhang Bin, An Investigation on the Users' Continuance Intention in Online Health Community__Based on Perceived Value Theory[J].Journal of Modern Information,2019,39(03):3-14+156.

[9] Sa Congmin,A Research of Brand, Advertising and Praise Influence on Purchase Intention Based on Customer Perceived Value Theory[D].South China University of Technology,2011.

[10] Wu Zhe, The Research of User Acceptance Model of Native Advertisement Based on UTAUT Theory[D].South China University of Technology,2017.

[11] Yang Chao, Research on the Factors Affecting Traffic Migration of Native Advertising[D].Jilin University,2019. 